\title{
The Application of Mongolian Cultural Symbols in the Design of Dynamic Character Emoticons
}

\author{
Suyalema Huo ${ }^{1}$ Baojun $\mathrm{Chu}^{1, *}$ Luoxuan Zhou ${ }^{1}$
}

\author{
${ }^{1}$ Xiamen Academy of Arts and Design, Fuzhou University, Xiamen, Fuzhou, China \\ *Corresponding author. Email: t17075@fzu.edu.cn
}

\begin{abstract}
This article uses emoticons as the research object, researches the integration of Mongolian national cultural symbols into the design of emoticon and conducts design practice. This article studies on creating a set of dynamic character emoticons that reflect Mongolian culture with Mongolian costumes, language, colors, etc. It provides theoretical basis for the design of emoticons rich in ethnic symbols by consulting literature and book materials, discusses the expression form of the Mongolian dynamic character emoticon design and the function and significance of spreading national culture, and conveys a more interesting Mongolian nation culture.
\end{abstract}

\section{Keywords: Mongolian culture, symbols, dynamic emoticons, design}

\section{INTRODUCTION}

The field of communication studies emoticons. These emoticons are transmitted by social platforms as nonverbal symbols in interpersonal communication on the Internet. In the era of digital media, dynamic expression has become the basic way of people's daily communication and interaction, such as: short videos, dynamic emoticons, dynamic text expressions, etc., which promote communication between people and affect people's thoughts and emotions, social life and cultural exchanges. Dynamic emoticons are also playing an increasingly important role in daily communication and cultural communication. This article analyzes the Mongolian clothing, colors, language and other symbols, applies them to the dynamic character emoticons, and explores the role and influence of the dynamic character emoticons in dynamic character expressions.

\section{OVERVIEW OF THE DYNAMIC EMOTICONS}

\section{A. Overview of the development of dynamic emoticons}

One of the oldest ways of expression in human society is to use pictures to express daily life and inner emotions. Since the Stone Age, humans have learned to express emotions and convey information through stone carvings and hieroglyphs. In 1982, Professor Scott Falman of Carnegie Mellon University in the United States entered the character ":-)" for the first time on an electronic bulletin board to represent a smile, and since then emojis have appeared. Since then, online emoticons have become popular all over the world. [1] . As real-time communication programs and forums began to use more vivid small patterns to express emotions, at the end of the 20th century, a new vocabulary (Emoticon) appeared in English, which cleverly combined "Emotion" and "icon" into a new word. [2] In today's society, with the development of the Internet, dynamic emoticons have become the most common way to communicate on social software such as WeChat and QQ. As of December 2017, the utilization rate of WeChat circles for Friends Moments and Qzone users was $87.3 \%$ and $64.4 \%$ respectively; as a social media, Weibo's user growth rate in 2017 was $40.9 \%$, an increase of $3.8 \%$ from December 2016. The utilization rates of Zhihu, Douban and Tianya-China have all increased. The user utilization rate is $14.6 \%$, $12.8 \%$ and $8.8 \%$, respectively. [3] In the process of communication, emoticons have already occupied a large part of the chat process. Many netizens often use various emoticons to express their feelings at the time. Today, the form of online emoticons has changed, including types, attributes, content, themes, and expressions [4].

\section{B. Current status of the use of dynamic emoticons}

According to Swyft Media's statistics, 2 billion smartphones worldwide send 6 billion emoticons and pictures every day. According to $\mathrm{WeChat}$ data, there are now 30,000 emoji packs and more than 70,000 emoji creators on the entire WeChat platform, and more than 600 million WeChat emoticons will be sent every day. The emoticons sent on BAT platform have reached hundreds of billion times in 2016. At present, the research of emoticons comes from linguistics, communication, sociology, psychology and other fields, mainly from the linguistic function of emoticons [5], communication meaning [6] and so on. According to 
the article "Research on the Phenomenon of the Spread of Online emoticons in Chinese Networks", the analysis of the survey data of emoticon users in terms of the use of crowds, use behaviors, and psychology of use reached the conclusion that college students are the most active when making emoticons. They not only have creative ideas, but also have various production techniques and use passion. However, more professionals in the workplace choose to use rational words to express themselves. As a result, elderly people are also affected and want to experience new ways of chatting and expression, and it is becoming popular to use emoticons in chats on various social platforms. [7]

\section{FEATURES OF DYNAMIC CHARACTER EMOTICONS}

\section{A. Universality}

Emoticons are mainly composed of visual symbols and text symbols. Its production is essentially a resymbolization of the original symbols and a practice of symbol reconstruction. [8] Darwin believed that facial expressions are natural, whether they are humans or animals, both express and recognize emotions in the same way. The emoticons are also connected, with the corners of the mouth rose pointing to the smiling face and the corners of the mouth pointing down to the crying face. Smiles, laughs, shy smiles, mischievous smiles, and smirks are universal and common. In addition to some common facial expressions, there are also facial expressions such as waving fingers hello or goodbye, clapping fingers for support, walking a few steps, bending waist and pointing to sorry or tired. These are what everyone often uses in daily life and chat. Emoticons use nonverbal symbols as the main method and use the trinity of images, text and emotions to express emotions.

\section{B. Convenience}

Convenience is reflected in the fact that people can convey their inner joy, anger, sadness and other emotions to the other party with a light touch of their fingers during the chat. It is simple and fast, and there is no need to type a string of words to waste time. The same is true for office workers. Because everyone has their own limitations, for example, children and the elderly can't type. When people chat with others, typing can feel like a waste of time, and some people can't write. In these cases, clicking dynamic emoticons are used to express emotions. The advantage is that it is convenient and fast, and can reach the realm of expressing emotions. For example: "Crying" emoticons can omit sentences such as "I'm really sad", "I'm sad" and other sentences. "Praying with both hands" emoticons can omit sentences such as "Praying for God's blessing", "Excuse me" and other sentences. "Angry" emoticons can omit the words "I'm angry",
"I'm very angry and going to get angry" and so on. Dynamic emoticons can show its convenience and change the atmosphere.

\section{Interestingness}

Compared with static emoticons, one of the biggest advantages of dynamic emoticons is its interestingness. Dynamic emoticons can show complete expressions and make expressions more vivid. They add a complete set of body movements and corresponding emoticons to the original only a certain freeze-frame expression, making the entire chat scene more relaxed and interesting. For example, the main feature of graffiti emoticons is "ugliness". The absurd, exaggerated, funny, and comical body is presented in a dramatic and interesting manner. Its creativity adds a different color to life, and is popular with children and young people. The number of users keeps increasing. For example, the exaggerated facial design of some dynamic emoticons makes the chat content more extreme. The combination of body movements and facial expression changes make up for the lack of context in the virtual chat environment. The dynamic emoticons add a relaxed and pleasant atmosphere to the chat process, and the chat is no longer constrained.

\section{Communicability}

Whether it is emoji or text symbols, they have one thing in common: communicability. Any kind of symbol comes from the purpose of communication and establishing interpersonal relationships. For example, when chatting with people for the first time, they will say to each other: "Hello!" and "Are you there? You are?" and sentences like this, but it is difficult to get a response. As the emoticons on WeChat, Weibo, QQ and other platforms become richer and closer to life and visualize, they have transformed from static images to dynamic images that can express content. The emoticons also make the communicative effect gradually increase. Playful and friendly dynamic expressions, body movements and facial expressions can stimulate people's desire to communicate through psychological mechanisms such as emotions. In addition to these features, the dynamic character emoticons also have the characteristics of fun and fashionable.

\section{EXPRESSION FORM OF MONGOLIAN DYNAMIC CHARACTER EMOTICONS}

\section{A. Design and application of clothing symbols}

Costumes play a representative role in each emoticon and an expression of identity. Due to the diversity of clothing symbols, the visual image of emoticons has gradually become rich and diverse. For example: at 11 o'clock on the morning of May 6, 2016, 
the first emoji with the theme of "National Unity and Family" created by the teachers and students of the School of Journalism and Communication of Communication University of China was officially released on the WeChat emoji platform. ("Fig. 1") The various cartoon characters in ethnic costumes in the emoticons are naive and ethnic. The "meng meng da (cute)" expression and body language expresses the common vocabulary such as "really good brother", "Yakxi", "safe journey", "no problem", "do it right away" and "copy that", which are widely popular on WeChat platform. It can be seen that clothing symbols play an important role in the dynamic character emoticons.

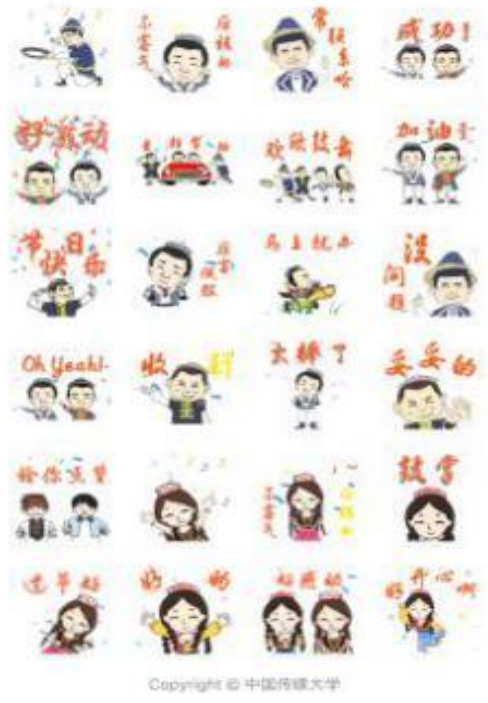

Fig. 1. A collection of facial emoticons for ethnic unity and a family.

The design of the character is crucial to the influence of the emoticons. Mongolian robes reflect Mongolian customs in terms of materials, crafts, styles, dressing methods and use processes. This article incorporates the elements of Mongolian robes, the traditional Mongolian costumes, into the design of the emoticons. The purpose is to spread Mongolian culture more widely and bring another way to express emotions for Mongolians. The style of Mongolian robes can not only reflect the curvaceous beauty of the human body, but also can reflect the generous, rude and frank character of Mongolian herders. The typical traditional Mongolian robe and Mongolian hat styles are extracted and combined with modern styles to give a brand new style. A complete, concise and generous Mongolian clothing style is applied to the design of the clothing image of the dynamic character emoticons. This method of designing clothing symbols is conducive to allowing the public to directly improve their preference for Mongolian clothing and fully understand it, so that more people have a good impression and deepen impression of Mongolian clothing, and it enables them to take the initiative to understand the unique humanistic feelings and ethnic costume culture of the Mongolian nationality. (See "Fig. 2")

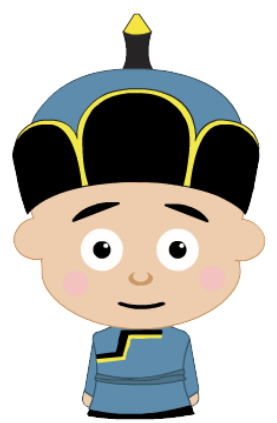

Fig. 2. A dynamic Mongolian character emoticon (smile).

\section{B. Design and application of national color symbols}

Vision is the most comprehensive and extensive way of feeling researched at present, and neither drawing nor product design can do without vision. [9] Thomas Young proposed the theory of color vision in 1800 . He advocated that the eyes of normal people have three types of color receptors, which can produce basic sensations for the three colors of red, green, and blue. Other colors are obtained by adding and subtracting these three colors. [9] According to this theory, color as a visual language has a strong visual impact. The color can fully express human emotions and consciousness. First of all, according to the psychological illusion, the physical characteristics of color are divided into "cold color" and "warm color". Red, orange, and yellow are warm color, while orange and red are extremely warm. Green and blue are cool colors, and blue is extremely cold. Cyan and purple distinguish between cool and warm color. Whether it is warm, cool or intermediate colors, they have their own unique characteristics. In the traditional Mongolian color concept, the four colors of blue, white, yellow and red are admired colors. White symbolizes the Mongolian people's simplicity, and kindness, and yellow is associated with the hero Genghis Khan, who holds a high status in their hearts and worships his heroic deeds. Red represents flame and passion. Mongolians have a soft spot for red. In this emoticon, the blue in the hearts of Mongolians in the warm color system is used as the main color and yellow is the auxiliary color. Blue, Mongolian called "Huhe", it represents the color of the Mongolian nationality. Mongolians advocate blue in all aspects of daily life and production activities, and of course, clothing is no exception. Dark blue is the main color of men's Mongolian robes, so the combination of dark blue and yellow is used to present the clothing color of the character's expression pack, enhance the visual effect, improve people's goodwill towards Mongolian colors, 
and can create different color atmosphere and effects in the chat process. (See "Fig. 3" "Fig. 4")

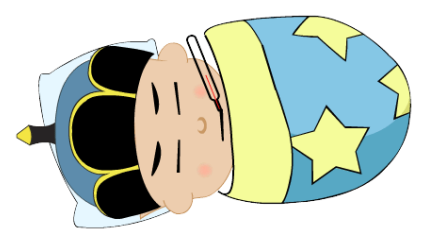

Fig. 3. Mongolian dynamic character emoticon (sleeping).

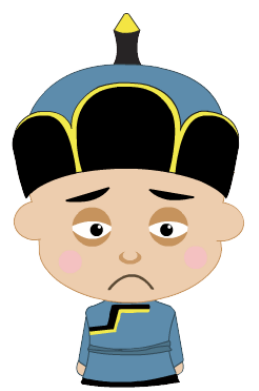

Fig. 4. Mongolian dynamic character emoticon (unhappy).

\section{Design and application of Mongolian language symbols}

As a medium, language plays an active role in the language and social foundation of people's identity. Language is not neutral, and people's understanding of reality and identity is inscribed in the language. [10] Mongolian is a language recognized by the Mongolian people. It is used to write Mongolian characters. Compared with other languages, Mongolian characters have different writing forms, reading methods and pronunciation arrangements. Mongolian has the characteristics of pure Pinyin writing, simple gestures, and quite deep social foundation. It is also a historical and cultural heritage of the Mongolian nationality. At the same time, it also has shortcomings such as vertical writing, the phenomenon of the same shape and different reading, and the specificity of the font that is not convenient to use international advanced technology. However, it is unique, and it is a noble and loving mother tongue in the hearts of Mongolians. In the dynamic emoticons, the single use of Mongolian characters to express the content to be presented will cause users to find it difficult to understand the meaning and reduce their interest. However, the combination of Mongolian and Chinese is used in the dynamic emoticons, which can more completely reflect the content of the emoticons and is beneficial to the Mongolian and Han people at the same time. For example, in the emoticons of crying and happiness in
"Fig. 5" and "Fig. 6", the Mongolian characters and Chinese are typeset together and placed on the upper right of the character, so that the receiver can easily and clearly understand the sender's thoughts and intention during the chat. This can better spread Mongolian language and culture and make more people be full of curiosity. People who are interested in Mongolian can also learn simple Mongolian writing and expression.

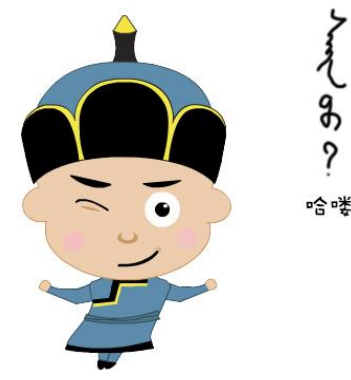

Fig. 5. Mongolian dynamic character expression pack (say hello).

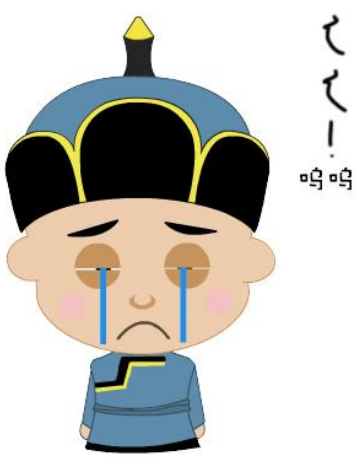

Fig. 6. Mongolian dynamic character expression pack (crying).

\section{THE CULTURAL COMMUNICATION FUNCTION AND SIGNIFICANCE OF DYNAMIC EMOTICONS}

The application and development of emoticons have brought new experiences to human communication. The dynamic character expression packs rich in Mongolian cultural symbols play a role in making up for the lack of context, enhancing the communicative effect, constructing an identity image, and spreading and inheriting Mongolian national culture in the process of chatting between people. When people communicate online, emoticons have become an important way for people to express their emotions, and there are many kinds of emoticons that people come into contact with. [11] The Mongolian cultural elements have also enriched the dynamic emoticons, which enables people to express their ideas more accurately, and adds more color emotions and interactive functions to simple text communication. Mongolian language, clothing culture, wedding customs, sacrificial rituals and daily Nadam 
are all unique cultural symbols of Mongolian nationality. Using these symbols to redesign the dynamic character emoticons can reflect different content and play more roles. As the most popular and down-to-earth communication carrier on today's online platforms, and as a form of spreading Mongolian culture, it has brought new ways to spread Mongolian culture so that it is no longer limited to communication modes such as books, entertainment and film and television.

\section{CONCLUSION}

In summary, as a popular communication carrier, dynamic emoticons are a new era of hieroglyphics and a basic tool for people to communicate on social networking platforms. However, the dynamic character emoticons are also gradually changing in image. The Mongolian cultural symbols are integrated into the dynamic character emoticon, giving it a new and unique image full of national colors. It also reflects the ethnic customs and culture of the Mongolian people, and can also promote exchanges between ethnic groups, enhance feelings, and make the country more united and friendly. This kind of chatting with national characteristics makes up for the shortcomings of social networking and the single atmosphere in the chat process, promotes the way of people's online communication, and spreads the national culture. Spreading national culture through dynamic emoticons can enable more people to understand Mongolian costume culture, color symbols and language culture, so that more people can understand it and learn and use it. Such dynamic emoticons with national characteristics also need continuous innovation and expansion to be applied to various chat platforms.

\section{References}

[1] Zhao Shuangying, Yao Wang, Emoji · Emotion - Plot: The Development and Evolution of Online Emoji [J]. Press Circles, 2013: 2. (in Chinese)

[2] Wei Zhuan, Zeng Meilin, Qu Changli, Network Emoji Design in Social Context [J]. Packaging World, 2015(03): 84-86. (in Chinese)

[3] China Internet Network Information Centre (CNNIC): The 41st Statistical Report on Internet Development in China, http://www.cac.gov.cn/2018 -01/31/c_1122347026.htm, 201801-31. (in Chinese)

[4] Gui Bin, Yang Xiaoping, Zhang Zhongxia, etc. Research on Building Lexicon for Sentiment Analysis Based on the Chinese Microblogging Smiley [J]. Journal of Beijing Institute of Technology(Natural Science Edition), 2014: 5. (in Chinese)

[5] Yu Guangwu, Qin Yun, A Study on Internet Emoticons from the Linguistic Perspective [J]. Academic Journal Graduate School Chinese Academy of Social Sciences, 2011 (1): 130135. (in Chinese)

[6] Cao Jin, Jin Dan, Theoretical Interpretation on the Communication Power of Internet Strong Language Memes [J].
Chinese Journal of Journalism \& Communication, 2016 (2): 37 56. (in Chinese)

[7] Ma Yang, Research on Communication Pheomenon of Current Network Expression Package in China [D]. Liaoning University, 2019: 15-22. (in Chinese)

[8] Lin Aijun, Zhang Bo, Emoticons as Words: Symbol Consumption and Sociological Reflections of Online Emoticons [J]. Modern Communication(Journal of Communication University of China), 2019, 41(08): 35-40. (in Chinese)

[9] Li Yanzu, Wang Mingzhi, chief ed. Design Art Psychology [M]. Liu Sha compile, Beijing: Qsinghus Unviersity Press, 2006.7.86. (in Chinese)

[10] (Canada) Michael Blake, Comparative Youth Culture: The Sociology of Youth Cultures and Youth Subcultures in America, Britain and Canada [M]. Meng Dengying, Bi Ruixin, trans, China Youth Press, 2017, p239. (in Chinese)

[11] Zhou Peng, Jiang Ru, Analysis on the Application of Inner Mongolia Folk Customs in the Design of Dynamic Emoticons [J]. Art Science and Technology, 2019, 32(01): 283-284. (in Chinese) 